$\mathbf{N}^{\circ} 7072$

CLINICAL

INNOVATIONS

\title{
Tooth autotransplantation as an alternative to dental procedure or replace missing teeth. Cases series.
}

Paternostro D, Lucas E, Llaquet M, Hernández-Alfaro F, Gargallo-Albiol J. International Master in Oral Surgery. International University of Cataluña.

\section{Abstract}

This technique was introduced by Apfel and Miller in 1950. Is a conservative treatment consisting in transplant one tooth to another as alternative to dental implants. Agenesis as well as the premature loss teeth due to trauma, caries, periodontitis or endodontic problems can be indication for transplantation. Successful protocol improves aesthetics, mastication, speech and arch integrity. The horizontal and vertical bone architecture of the recipient site, it's delimiting factor as well as the anatomy of the donor tooth, which determine if it's possible to perform this procedure. According to the literature, this technique has been performed in tooth with partial root formation, and complete root formation, giving excellent results in long term according to dental implants and with different advantages and disadvantages.

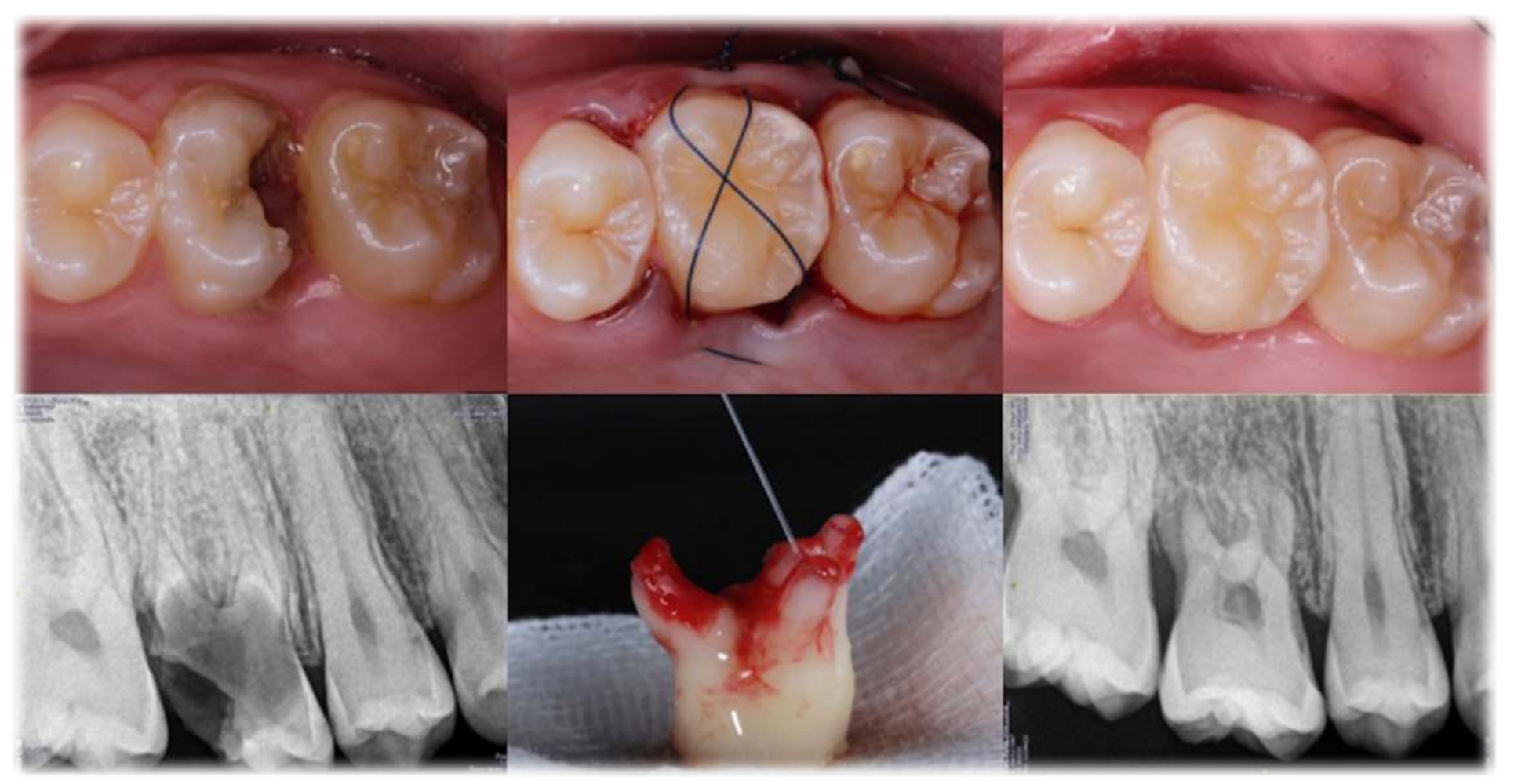

Background and Aim

The aim of this study it's to evaluate the outcoming of the autotransplantation technique in five (5) patients, performed by the same oral surgeon, and rehabilitated by the same endodontic specialist. And comparing the results with dental implant treatments.

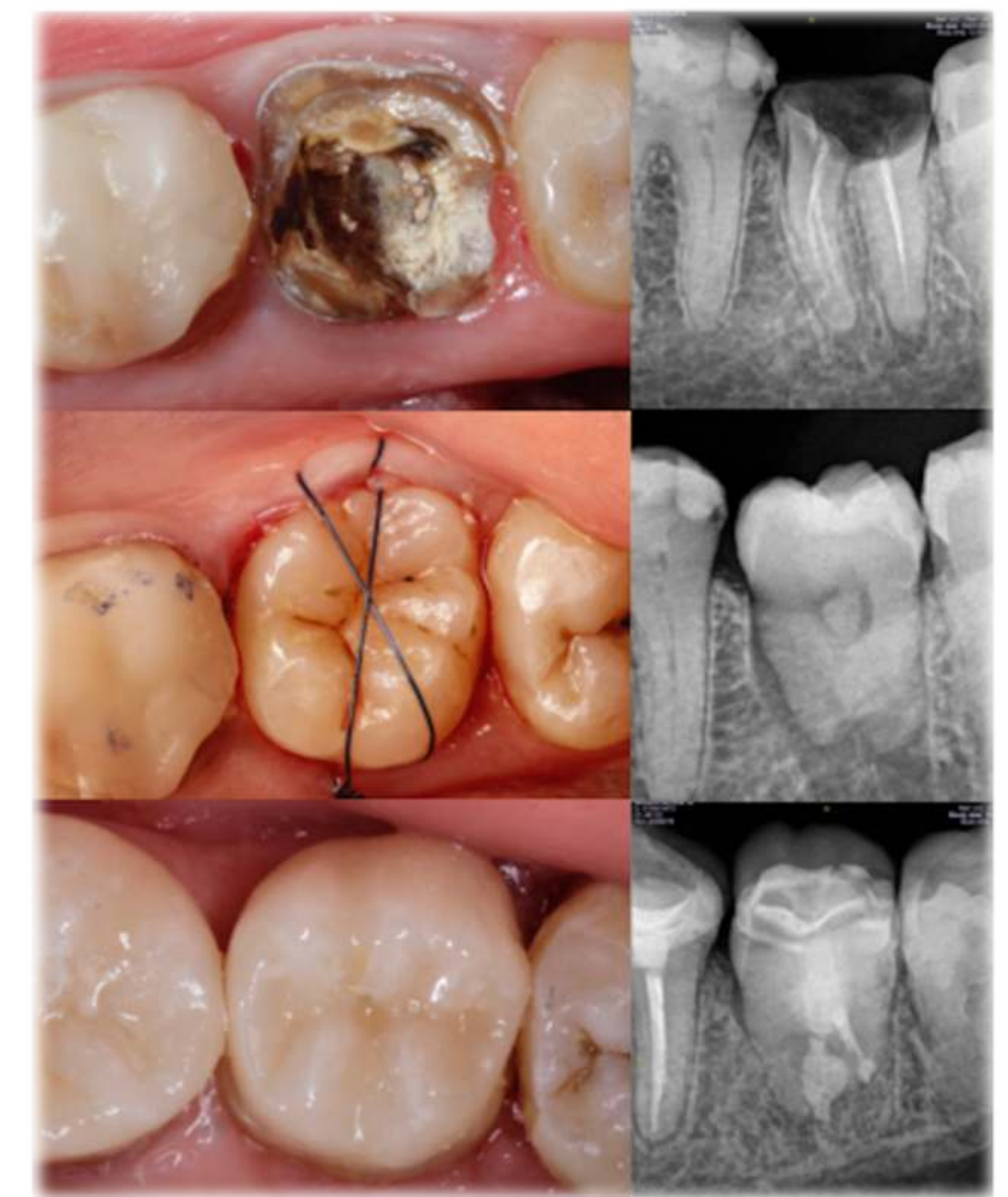

Methods and Materials

- Five patients were treated, in which an autotransplantation was performed per patient.

- Through the CT and periapical radiographs prior to surgery, the anatomy and dimension of the roots, mesio distal space and alatine or lingual vestibule of the tooth to be extracted and the tooth to be transplanted were evaluated.

- The extractions were atraumatically maintaining the architecture of the recipient bed and maintaining as much as possible the periodontal ligament. After the surgery was $600 \mathrm{mg}$ ibuprofen and rinses with $0.12 \%$ chlorhexidine

- Each transplanted tooth was splinted with 4-0 monofilament or orthodontic arch sutures for 14 days. After the 30 days, the treatment of sealed ducts with gutta percha in closed apices.

- As a last step, CAD-CAM overlays were made and cemented with resin cement.

\section{Results}

After one year follow up, in each clinical case excellent stability, preservation of alveolar architecture and aesthetics were obtained.

There were no postoperative complications or treatment failures No statistical alteration of probing pocket depth, architecture of the recipient side was stable in time indicating no marginal bone oss and keratinized mucosa.
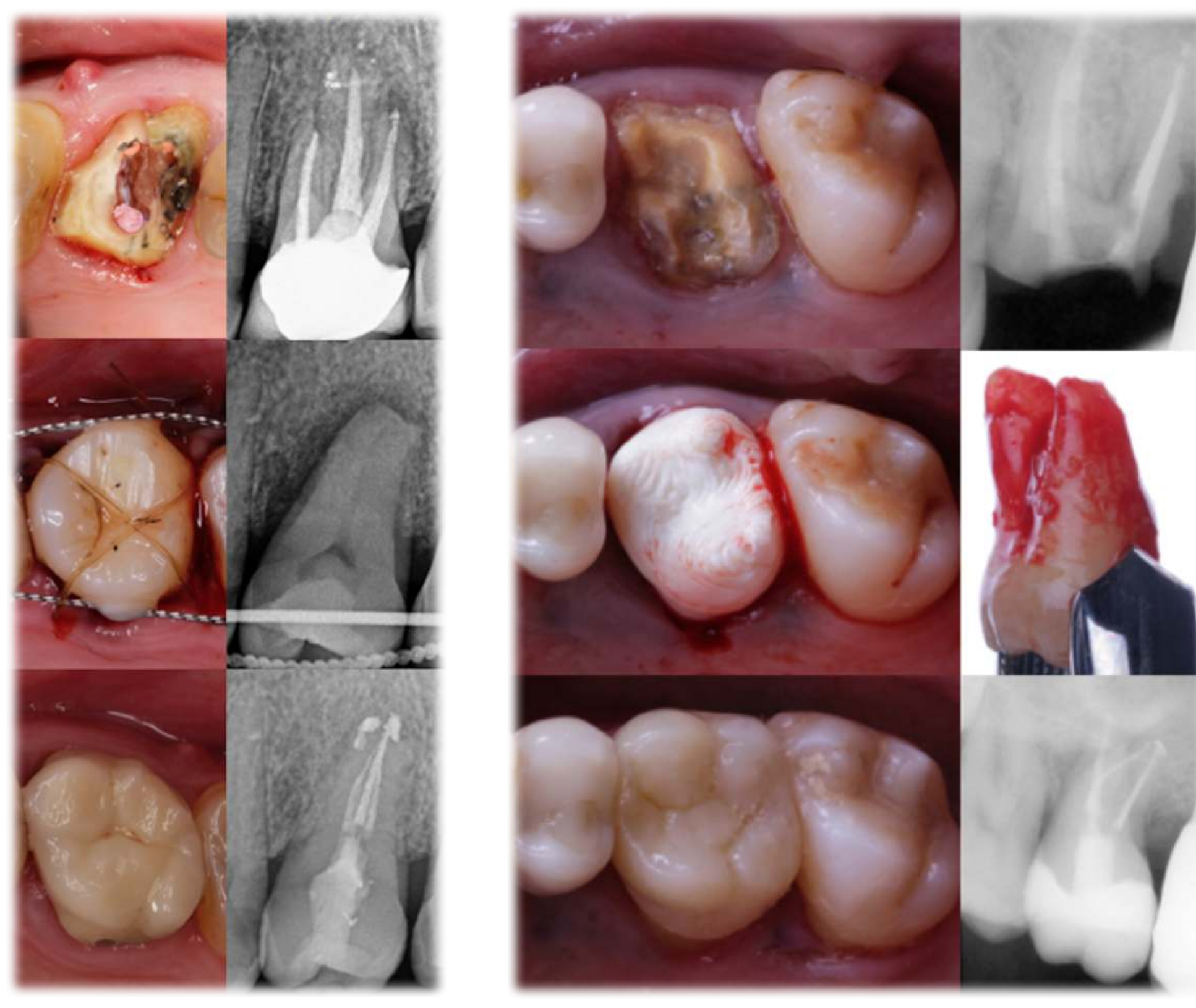

\section{Conclusions}

Dental autotransplantation is a sensitive technical procedure, in which much of the success rate comes from the skill and handling of the specialist's tissues.

Dental autotransplantation has excellent results preserving alveolar anatomy and obtaining high prosthetic aesthetics in relation to dental implant treatment solutions, with reducing costs and no contraindications in growing patients.
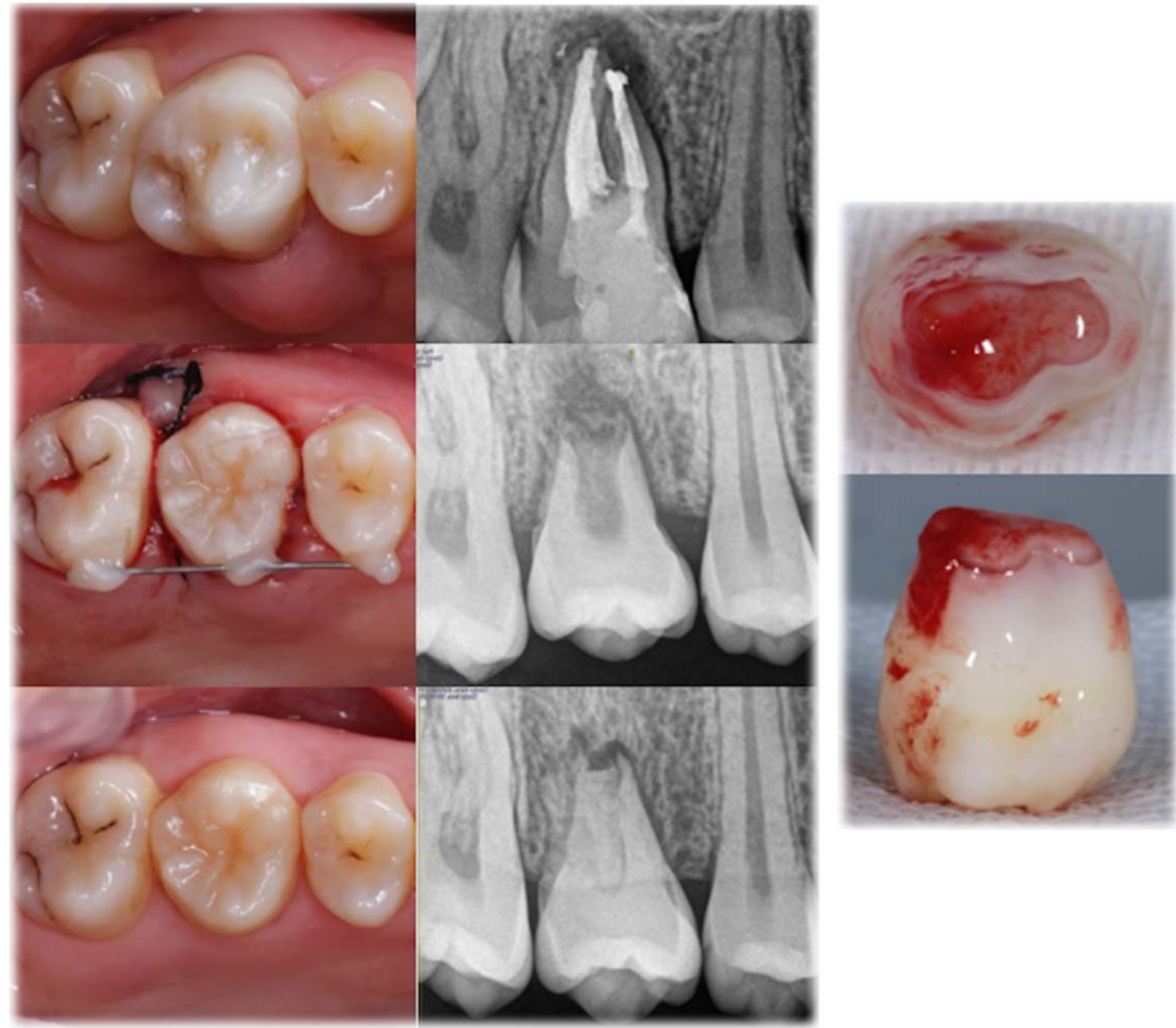

\section{References}

Autoplasty of Enucleated Prefunctional Third Molar Tooth, Oral Surgery, Oral Medicine, Oral Pathology. Autotransplantation of third molars with completely formed roots into surgically created sockets and fresh Autoplastic tooth transplantation: Its clinical applicability. Report of two cases of different types. Ora Surgery, Oral Medicine, Oral Pathology.

Retrospective study of 100 autotransplanted teeth with complete root formation and subsequen orthodontic treatment. American Journal Of Orthodontics \& Dentofacial Orthopedics.

Retrospective long-term evaluation of autotransplantation of premolars to the centra incisor revion Outcomes of autotransplanted teeth with complete root formation: a systematic review and meta-analysis. 